\title{
Optimization of the available spectrum of a WDM sensors network using a mode locked laser
}

\author{
M. Fernandez-Vallejo, D. Ardanaz and M. Lopez-Amo, Senior Member, IEEE
}

\begin{abstract}
This work experimentally proposes a new interrogation method which consists on the combination of the information given by both domains: time and frequency. On the one hand, the sensor information is obtained in the frequency domain as usual and, on the other hand, the detection of the different pulses generated through mode locking allows the identification of each FBG. It has been proved that FBGs spaced less than $0.15 \mathrm{~nm}$ can be interrogated. The sensor network based on simple ring Erbium doped fiber laser using the FBGs, located in a serial configuration, as wavelength selective reflectors for the lasing structure, acting them also as the sensing transducers elements.
\end{abstract}

Index Terms-Fiber Bragg gratings, multiplexing, networks, optical sensing, fiber lasers.

\section{INTRODUCTION}

$F_{c a n}$ BER optical sensor networks based on fiber Bragg Gratings (FBGs) have proven to be a powerful tool in the field of Structural Health Monitoring (SHM). Thanks to the advantages of FBGs and the versatility of fiber optical sensor networks, this technology is able to provide a solution for many applications: from large scale structures built by the human action such as tunnels, dams, bridges, piles, pipelines, railways; to large natural environments or natural events like tsunamis or geodynamical movements. [1-4]

The versatility of FBG sensor networks is associated with the design process due to the fact that there is a wide range of possibilities to combine. The aspects, in general, to take into account are the following: the network topology [5,6]; the use, or not, of optical amplification [7,8]; the operation mode of the network which can be based on a laser or not [9]. But in addition to these aforementioned facets, in the definition of a real project the cost is a fundamental issue. In this context, the wavelength multiplexing capability of FBGs is crucial because it reduces the price since only one optoelectronic unit is used for the whole network [10].

Regarding the operation mode, the fiber optical network based on a fiber laser offer an appealing improvement compared with the non-lasing ones: higher signal to noise ratio (SNR) because the noise associated with the amplification is

Manuscrited submitted May 2015. This work was supported in part by the Spanish Government projects TEC2013-47264-C2-2-R, innocampus and FEDER funds.

M. F-V, D. A and M. L-A. authors are with the Department of Electric and Electronic Engineering, Public University of Navarra, Pamplona, Spain. (corresponding author to provide Montserrat.fernandez@unavarra.es) reduced by the lasing process. This behavior enhancement has opened up new opportunities such as the multiplexing of many sensors or the development of long-range networks. Therefore, provided that a lasing structure is considered, the inclusion of optical amplification is compulsory: Erbium doped fiber amplification (EDFA) or Raman amplification are the most common options, each one with its strengths and weaknesses. Regarding EDFAs, they offer a useful gain bandwidths, high pumping efficiency, low intrinsic noise and low gain dependence on light polarization. EDFAs gain, however, is not uniform over the whole bandwidth and it is characterized by homogeneous broadening. And with respect to Raman amplifiers, Raman gain mechanism is intrinsic to the silica fiber, which means, no specialty fiber is needed; Raman amplifiers can achieve gain at any wavelength by choosing an appropriate pump wavelength; so the bandwidth may be extended simply combining multiple pump wavelengths. Nevertheless, Raman amplifiers have relatively poor pumping efficiency at low signal powers and long fibers are required. Thus, the kind of light source employed in the system is going to determine the number of multiplexed FBGs because it establishes key aspects such as the optical signal to ratio (OSNR) and the bandwidth.

But, not only does the light source impose limitations, the FBGs themselves require distinct wavelength band to allocate each sensor and also a safety bandwidth to avoid crosstalk between adjacent sensors when the FBGs-based systems operate in continuous-wave mode [11,12]. Usually, the wavelength spacing between channels is about $0.4 \mathrm{~nm}$.

Taking into account that multiplexing capability is of prime importance when real sensor networks are developed, other interrogation systems based on other sources must be investigated to overcome the actual limitations. The general requirements for an ideal interrogation method are as follows: they must provide high sensitivity to Bragg wavelengths shifts, large measurement ranges, immunity to optical power fluctuations, low environmental sensitivity, compatible with sensor multiplexing, simple and low cost [13]. There are two types of mode-locked fiber lasers (MDFL): actively-MDFLs which use an internal modulation; and passively-MDFL which are based on non-linear effects or saturable absorbers. Saturable absorbers turn the continuous wave output intro ultra-short pulse operation [14]. The proposed solution is based on an Erbium doped fiber lasers which obtains the ultrashort pulses through a passively-MDFL. Although these pulsed lasers sources have been studied in depth to generate ultrashort pulses [15-17], few works, up to now, use them in FBG sensor systems [18-20].

In this work, we propose and demonstrate the interrogation of FBGs spaced less than $0.15 \mathrm{~nm}$. The FBG sensor network is 
based on an Erbium doped fiber laser and the interrogation method consists on the combination of the information given by both domains: time and frequency. On the one hand, the detection of the different pulses generated through mode locking allows the identification of each FBG and, on the other hand, the sensor information is obtained in the frequency domain as usual

\section{EXPERIMENTAL SET-UP}

The proposed sensor system for multiplexing pairs of FBGs and based on a simple Erbium doped fiber laser can be seen in Fig. 1. As in any sensor system, it can be divided into two different parts: the sensor unit itself, which includes the serial array of FBGs, and the monitoring station, which consists of the electro-optical components.

The sensing unit is composed of 4 pairs of FBGs and a referenced FBG. The pairs of FBGs are centered in 1544.12 $\mathrm{nm}\left(\lambda 1\right.$ and $\left.\lambda 1^{\prime}\right), 1545.594 \mathrm{~nm}\left(\lambda 2\right.$ and $\left.\lambda 2^{\prime}\right), 1547.116$ ( $\lambda 3$ and $\left.\lambda 3^{\prime}\right) \mathrm{nm}$ and $1550.154 \mathrm{~nm}$ ( $\lambda 4$ and $\left.\lambda 4^{\prime}\right)$. The center wavelength of a pair of FBGs, however, is not exactly the same due to the fabrication process, thus, the higher difference in the worst case is of $0.072 \mathrm{~nm}$. The bandwidth of all the FBGs is $0.18 \mathrm{~nm}$ and their reflectivity's are around $95 \%$. The spatial separation of two contiguous FBGs of a same subarray is around $2 \mathrm{~m}$, while the spectral separation is approximately $1.5 \mathrm{~nm}$. Taking into account the FBGs are located in a serial configuration, it is considered to locate a reference FBG $\left(\lambda_{\text {ref }}\right)$ at the end of the array in order to corroborate there is no a breakage in the fiber or a FBG does not work. As the operation mode of the sensor system is based on a fiber laser, the FBGs play simultaneously a dual-role: on the one hand, they are wavelength selective reflectors for the lasing structure and, on the other hand, as the sensing transducers elements. As the picture shows, the FBGs are connected to the transmission channel through a circulator which inserts the FBGs' reflected signals into the ring, ensuring unidirectional operation and therefore avoiding undesired effects such as spatial hole-burning (SHB).

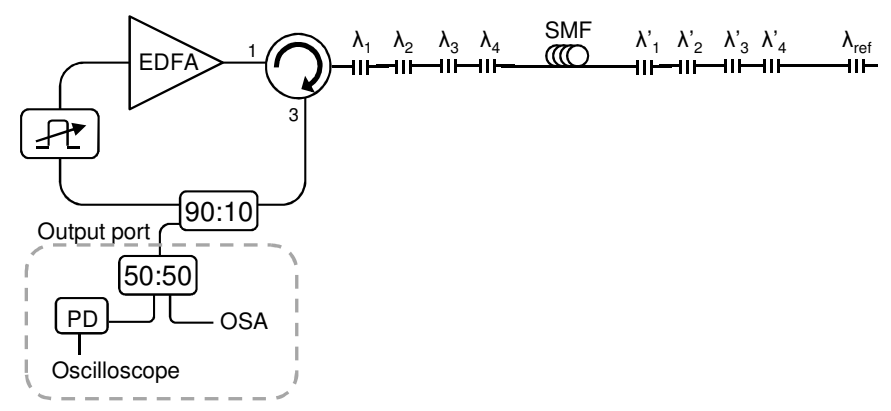

Fig. 1. Experimental set-up for the proposed sensor system for multiplexing pairs of FBGs. PD: photodetector. OSA: Optical spectrum analyzer. SMF: Single mode fiber. EDFA: Erbium doped fiber amplifier

Secondly, regarding the monitoring station, it consists of: a commercial EDFA from MPB technologies which provides the gain; a programmable tunable filter; a circulator to connect the monitoring station with the sensing unit, and a 90:10 coupler to extract $10 \%$ of the laser output power. The programmable tunable filter is a device from Finisar and the model is Wave Shaper 1000S. The main characteristics for this experimental work are the following: insertion loss $6.5 \mathrm{~dB}$; filter bandwidth $10 \mathrm{GHz}$ to $5 \mathrm{THz}$; bandwidth setting resolution $1 \mathrm{GHz}$; settling time $500 \mathrm{~ms}$. The programmable tunable filter allows to select its filter bandwidth from 40 to $0.08 \mathrm{~nm}$ and its filter center wavelength with a precision of $8 \mathrm{pm}$ in the communications C Band (1527.4-1567.4 nm). Finally, the output port is connected through a 50:50 coupler to the detection devices: an oscilloscope with a resolution of $1 \mathrm{~ns}$ and an optical spectrum analyzer (OSA).

The operation mode of sensor system is based on an EDFA fiber laser, thus lasing will take place provided that the gain in the cavity overcomes the total cavity losses. The tunable filter sweeps in wavelength and select which FBG is going to lase at each time. This device has a key function: it avoids the multiwavelength operation and, therefore, problems regarding competition gain characteristic of Erbium doped fiber amplifiers are eliminated. For these reasons, the FBG's serial configuration is not an obstacle.

The main novelty of the proposed sensor system is related to the interrogation system. As Fig. 1.shows, the array of FBG sensors can be divided into two sub-arrays spaced by 6 meters of single mode fiber (SMF) and each sub-array includes a FBG of each pair. The section of $6 \mathrm{~m}$ long between the two groups is only to simulate a real scenario, but this length is arbitrary. And the spatial separation between two contiguous FBGs is $2 \mathrm{~m}$. When the tunable filter wavelength sweeps in wavelength, there are three different situations:

- The easiest case, when the wavelength of the tunable laser does not matches with any FBG: there is no laser condition in the cavity.

- The worst case, when the wavelength of the tunable laser overlaps with the center wavelength of one FBG, but, due to external factors, the other FBGs have slightly a different center wavelength. In this case, when the tunable laser sweeps, the OSA shows two spectra slightly different in center wavelength. But, due to the interrogation system proposed, it is possible to recognize if the information comes from the FBG of the first subarray $\left(\lambda_{1}, \lambda_{2}, \lambda_{3}\right.$ or $\left.\lambda_{4}\right)$ or from the FBG of the second subarray $\left(\lambda_{1}{ }^{\prime}, \lambda_{2}, \lambda_{3}{ }^{\prime}\right.$ or $\left.\lambda_{4}{ }^{\prime}\right)$. The method is based on the analysis of the pulses generated through mode locking in the cavity and detected with the oscilloscope. These pulses obey the following equation:

$$
T=\frac{n_{g} L}{c}
$$

Where: $\mathrm{n}_{\mathrm{g}}$ is the mode's group index, $\mathrm{L}$ is the length of the cavity and $\mathrm{c}$ is the light speed in the vacuum.

Taking into account the previous equation, once two pulses are detected with the oscilloscope, it is possible to obtain easily the length $\mathrm{L}$ and, therefore, associated with this length; it is possible to establish the FBG that is being monitored.

- Finally, an intermediate scenario, when the wavelength of the tunable laser is coincident with the reflection band 
of one the pairs of FBGs and both FBGs have exactly the same center wavelength. In this case, the reflected wavelength observed in the OSA belongs to the FBG of the first subarray $(\lambda 1, \lambda 2, \lambda 3$ or $\lambda 4)$. Only to confirm this assertion, as in the previous case, the information of the pulses in the oscilloscope can be analyzed.

\section{EXPERIMENTAL RESULTS AND DISCUSSION}

This section details how the proposed interrogation system was tested in order to check its viability. This process involves analyzing thoroughly which is the minimum difference between the wavelength centers of a pair of FBGs the interrogation system is able to detect.

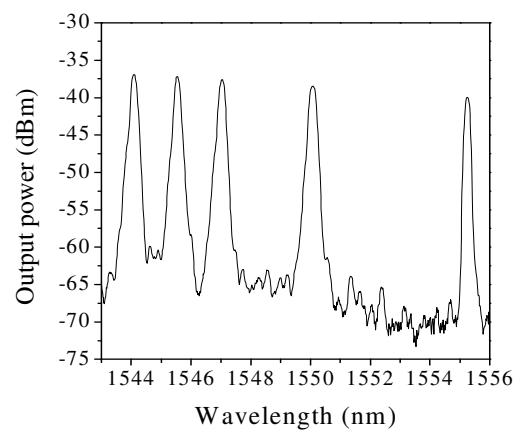

Fig. 2. Spectrum of the pairs of FBGs when they are at room temperature and the referenced signal (OSA resolution $0.1 \mathrm{~nm}$ )

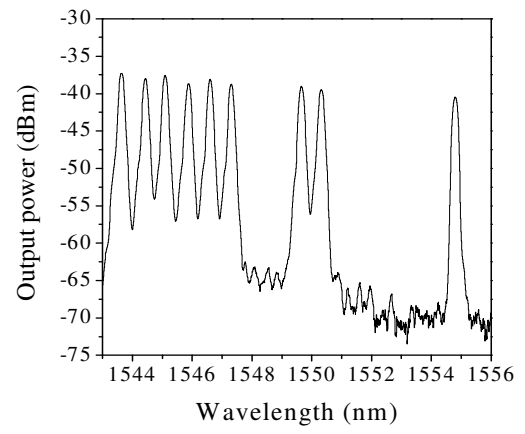

Fig. 3. Spectrum of the pairs of FBGs when the second group of FBGs are subject to temperature changes and the referenced signal (OSA resolution 0.1 $\mathrm{nm})$

When the FBGs are at room temperature and the tunable filter, with a bandwidth of $8 \mathrm{pm}$, makes a complete wavelength sweep, the obtained spectrum of the pairs of FBGs and the referenced signal is as Fig. 2. shows. It is obvious that using a conventional interrogation system based only on the wavelength center of the FBGs, it is not possible to distinguish if the obtained information comes from the first FBG or from the second FBG of a pair.

The procedure starts subjecting the FBGs to temperature changes. When the second subarray of FBGs are inserted in a climatic chamber and the temperature reaches $90{ }^{\circ} \mathrm{C}$, the spectrum of the pairs of FBGs is as Fig. 3 depicts where eight channels are clearly observed. The problem starts when the temperature decreases and the eight channels assigned to each FBG become only four channels. For this reason, it is proposed to use the pulses which are generated through mode locking and are inherent to any EDFA laser.

In a laser cavity, multiple longitudinal modes oscillate independently, and the output is a composition of random components, giving an averaged constant power output. However, if all longitudinal modes are in phase, the coherent sum of all longitudinal modes leads to an optical train of pulses, with a period equal to the cavity trip time. Fig.4. shows the pulses generated. As each FBG generates a different cavity length, the distances between pulses in each case is also unlike. This characterization of the laser cavity in the time domain allows to distinguish the measured data of two FBGs whose wavelengths are as close as $0.15 \mathrm{~nm}$. If the spectral FBG separation is lower than $0.15 \mathrm{~nm}$, the second FBG of each pair is not able to lase due to the first FBG capture most of the power and the emission lines will belong to the FBGs of the first subarray. For the first pair of FBGs, Fig.4 (a) depicted that the pulses are separated 230.72 ns for the FBG allocated in the first subarray; while $387.35 \mathrm{~ns}$ for the FBG allocated in the second subarray. Fig. 4 (b) illustrates the results for the last pair of FBGs, obtaining pulses separated 311.42 ns and 467.55 ns respectively for the first FBG of the pair and for the last whose wavelength centers. Taking into account this repetition rate of the pulses, it is possible to identify without ambiguity the position of each FBG: in the first case and considering the equation (1), the total cavity length is $48 \mathrm{~m}$, corresponding $46 \mathrm{~m}$ to the ring length and being located the first FBG in the array at $1 \mathrm{~m}$ from the circulator.
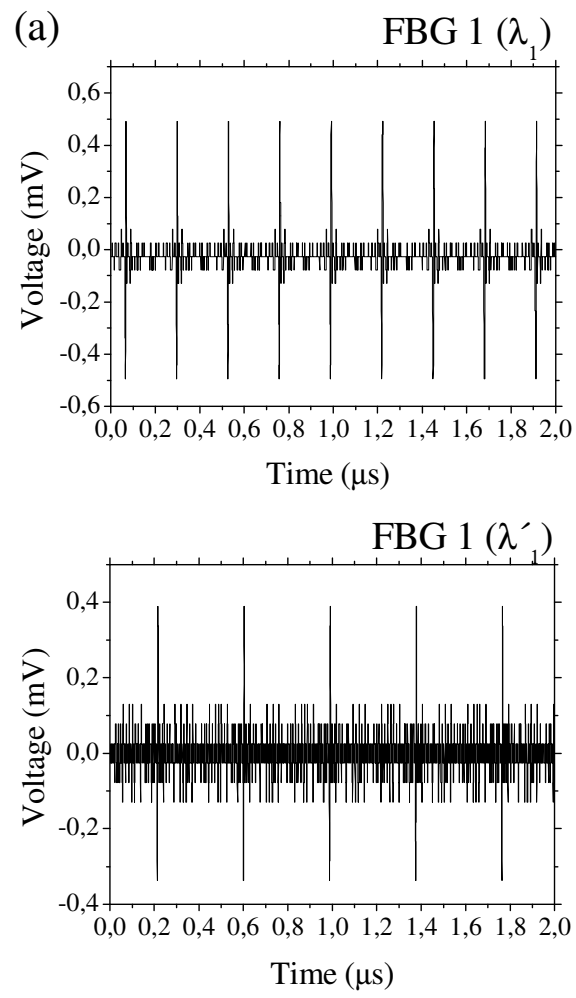
(b)
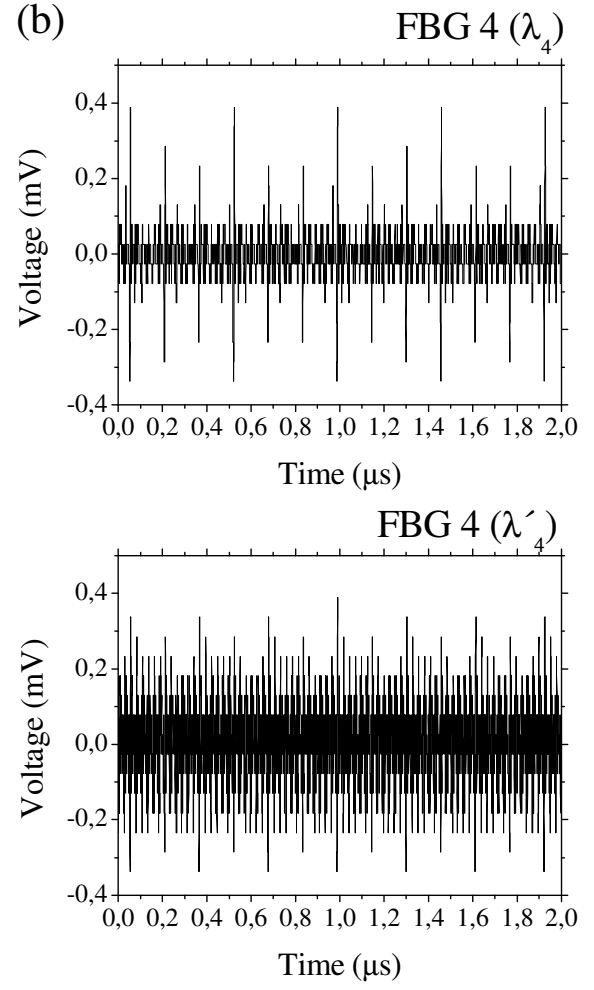

Fig. 4. Pulses generated by the cavity created with (a) the first pair of FBGs $\left(\lambda_{1}\right.$ and $\left.\lambda_{1}{ }^{\prime}\right)$ and (b) the last pair of FBGs $\left(\lambda_{4}\right.$ and $\left.\lambda_{4}{ }^{\prime}\right)$

In order to assess the sensing capability of the system, the FBG of the second subarray centered at $1547.07 \mathrm{~nm}$ was located in a climatic chamber and heated up. Figure 5 illustrates the linear behavior of the $\mathrm{FBG}$ versus the temperature obtaining a sensitivity of $10.7 \mathrm{pm} /{ }^{\circ} \mathrm{C}$. Taking into account that the information is encoded in the resonant wavelength, it is therefore considered appropriate to evaluate the wavelength stability of the EDFA fiber laser during 30 minutes. The instability is defined as the wavelength variation for a given interval of time and a specific confidence interval (CI), given as a percentage. The confidence interval (CI) is the estimated range of values where the parameter of interest is included [22]. Fig. 6. shows the results using a Brillouin optical spectrum analyzer, which offers a resolution of 0.08 $\mathrm{pm}$. Thus, the wavelength stability is $3.5 \mathrm{pm}$. Differences of sensing capabilities among FBGs are negligible, for this reason, we considered enough to include only one study to illustrate the sensing capability of the system and the linear behavior of the FBGs.

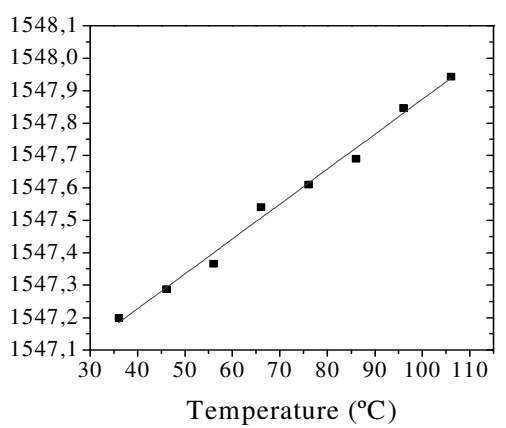

Fig. 5. Wavelength FBG evolution versus temperature

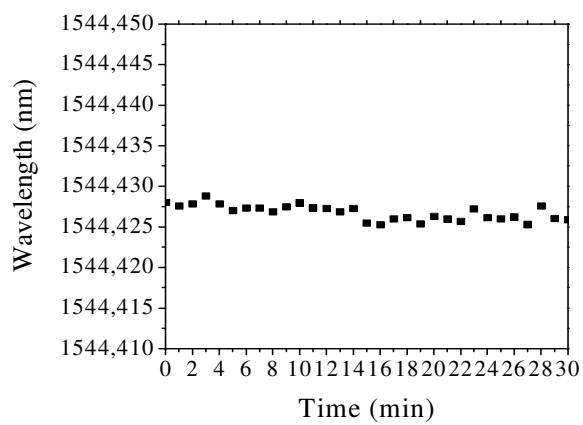

Fig. 6. Wavelength stability

\section{CONCLUSIONS}

This work experimentally demonstrates that pairs of FBGs allocated in a serial array and spectrally spaced less than 0.15 $\mathrm{nm}$ can be interrogated using a mode locked laser. The sensor network is based on an Erbium doped fiber laser. The interrogation method consists on the combination of the information given by both domains: time and frequency. On the one hand, the sensor information is obtained in the frequency domain as usual and, on the other hand, the detection of the different pulses generated through mode locking allows the identification of each FBG. The train of pulses allows the identification, without ambiguity, of the position of each FBG in the sensor network. The FBGs, located in a serial configuration, are the wavelength selective reflectors for the lasing structure and the sensing transducers elements at the same time. The intra-cavity tunable filter enables the multiplexed operation. Finally, in order to validate the operation of the FBG sensor system, the sensors arrays were heated up in a climatic chamber. Individual response of an FBG located in a climatic chamber for heating, was observed to be linear, with a slope of $10.7 \mathrm{pm} /{ }^{\circ} \mathrm{C}$. To sum up, with this technique, an efficient utilization of the spectrum is achieved, thus it is possible to increase the number of FBGs that can be multiplexed using a single interrogation light source.

\section{REFERENCES}

[1] H. Li, D. Li and G. Song, "Recent applications of fiber optic sensors to health monitoring in civil engineering," Eng. Struct., vol. 26, n. 11, (2004), pp. 1647-1657.

[2] M. Majumder, T. K. Gangopadhyay, A.K. Chakraborty, K. Dasgupta, D.K. Bhattacharya, "Fibre Bragg gratings in structural health monitoring-present statusand applications," Sens. Actuat. A Phys., vol. 147 , n. 1, (2008), pp. 150-164. 
[3] P. Ferraro, G. De Natale, "On the possible use of optical fiber Bragg gratings as strain sensors for geodynamical monitoring," Optics and Lasers in Engineering, vol. 37, n. 2-3, (2002), pp.115-130.

[4] C. Wei, C. Lai, S. Liu, W.H. Chung, T.K. Ho, H. Tam, S.L. Ho, A McCusker, J. Kam and K. Y. Lee, "A fiber Bragg grating sensor system for train axle counting," IEEE Sens. J., vol. 10, n. 12, (2010), pp. 19051912.

[5] J. Hu, Z. Chen, X. Yang, J. Ng and C. Yu, "100-km long distance fiber Bragg grating sensor system based on erbium-doped fiber and Raman amplification," IEEE Photonic. Technol. Lett., vol. 22, n. 19, (2010), pp. 1422-1424.

[6] S. Diaz, S. Abad, and M. Lopez-Amo, "Fiber-optic sensor active networking with distributed erbium-doped fiber and Raman amplification," Laser Photonics Rev., vol. 2, n. 6, (2008), pp. 480-497.

[7] M. Fernandez-Vallejo and M. Lopez-Amo, "Optical fiber networks for remote fiber optic sensors," Sensors, vol. 12, n.4, (2012), pp. 3929-3951.

[8] S. Abad, M. López-Amo, J.M. López-Higuera, D. Benito, A. Unanua, and E. Achaerandio, "Single and double distributed optical amplifier fiber bus networks with wavelength-division multiplexing for photonic sensors," Optics Letters, vol. 24, n. 12, (1999), pp. 805-807.

[9] M. Fernandez-Vallejo, S. Díaz, R. A. Perez-Herrera, D. Passaro, S. Selleri, M. A. Quintela, J. M. L. Higuera, and M. Lopez-Amo, "Resilient long-distance sensor system using a multiwavelength Raman laser," Meas. Sci. and Technol., vol. 21(094017), (2010).

[10] M. Bravo, M- Fernandez-Vallejo, M. Echapare and M. Lopez-Amo, "Multiplexing of six micro-displacement suspended-core Sagnac interferometer sensors with a Raman-Erbium fiber laser," Optics Express, vol. 21, n. 3, (2013), pp. 2971-2977.

[11] D. B. Huff and M. S. Lebby, "Fiber optic sensing technology: emerging markets and trends," in Proceedings of SPIE 6619, Naples, Italy, 2007, pp. 661902

[12] S. Abad, M. López-Amo and I.R. Matías, "Active Fibre Optic Sensor Networks," in Handbook of optical fibre sensing technology, J. M. Lopez-Higuera, ed., New York.

[13] J. L. Santos and W. N. MacPherson, Hand Book of Optical Fiber Sensing Technology, Chapter 18 Fiber Bragg Grating Interrogation Techniques, pp. 379-402, Ed. by J. M. Lopez-Higuera, 2002.

[14] Z. Wang, L. Zhan, J. Wu, Z. Zou, L. Zhang, K. Qian, L. He, And X. Fang, "Self-starting ultrafast fiber lasers mode-locked with alcohol," Optics Letters, Vol. 40, N. 16, (2015), pp.3699-3702.

[15] H. Hundertmark, D. Kracht, M. Engelbrecht, D. Wandt, C. Fallnich, Stable sub-85 fs passively mode-locked erbium-fiber oscillator with tunable repetition rate, Opt. Express 12 (2004) 3178-3183.

[16] D. Y. Tang and L. M. Zhao, "Generation of 47-fs pulses directly from an erbium-doped fiber laser," Optics Letters, vol. 32, n. 1, (2007), pp. 4143.

[17] X. Li, W. Zou, G. Yang, and J. Che, "Direct Generation of $148 \mathrm{~nm}$ and 44.6 fs Pulses in an Erbium-Doped Fiber Laser," IEEE Photonics Technology Letters, vol. 27, n. 1, (2015), pp. 93-96.

[18] Y. Wang, W. Lie, J. Fu and D. Chen, "Quasi-distributed fiber Bragg grating sensor system based on a Fourier domain mode locking fiber laser," Laser Physics, vol. 19, n. 3, (2009), pp. 450-454.

[19] B. C. Lee, E-J Jung, C-S Kim and M. Y. Jeon, "Dynamic and static strain fiber Bragg grating sensor interrogation with a $1.3 \mu \mathrm{m}$ Fourier domain mode-locked wavelength-swept laser," Meas. Sci. Technol., vol. 21(094008), (2010), pp. 1-5.

[20] C. Lavieja, S. Jarabo, M. Marin-Doñagueda, I. J. Sola, "Mode-locked erbium-doped fiber lasers as source for optical sensor networks over C and L bands," Optical Fiber Technology, Vol. 19, (2013), pp. 476-481.

[21] J.J. Fu, W. S. Liu, D. Chen and S. He, "Ultra-long-distance FBG sensor system based on spectrum-limited Fourier domain modelocking fibre laser with Raman pumps," Electronics Letters, vol. 44, n. 16, (2008), pp. 61-962.

[22] M. Fernández-Vallejo, S. Diaz, R. A. Pérez-Herrera, R. Unzu, M. A Quiniela, J. M. López-Higuera and M. López-Amo, "Comparison of the stability of ring resonator structures for multiwavelength fiber lasers using Raman or Er-Doped fiber amplification," IEEE J. Quant Electron., vol. 45, n. 12, (2009), pp. 1551-1557
Montserrat Fernández-Vallejo was born in Navarra, Spain, in February 1983. She received the M.Sc. degree in telecommunication engineering from the Public University of Navarra, Spain in 2008. In 2012 she received the Ph.D. degree from the Public University of Navarra, Navarra, Spain. During this period, she was a visiting Ph.D. student at the department of information engineering at Parma University, Italy, and at the Institute of Photonic Technology IPHT, Germany.

In 2014, she became an Assistant Professor in the Electrical and Electronic Engineering department of the Public University of Navarra.

Her research is focused on Raman amplifiers, erbium-doped amplifiers, fiber-optic sensors and multiplexing architectures

Diego Ardanaz was born in Navarra, Spain, in November 1984. He received the telecommunication engineering degree from the Universidad Publica de Navarra, Spain in 2015 and. during 2014 and 2015, he worked in the Optical Communications Group, Department of Electrical and Electronic Engineering from Universidad Publica de Navarra. His research interests are in fiber optic lasers, optical amplifiers, optical fiber sensor networks and multiplexing architectures.

Manuel López-Amo (M'91, SM '98) was born in Madrid, Spain, in 1960. He received the telecommunications engineering degree and Ph.D. degree from the Universidad Politécnica de Madrid, Spain in 1985 and 1989, respectively. From 1985 to 1996, he belonged to the Photonic Technology Department of the Universidad Politécnica de Madrid, where in 1990 he became an associate professor.

In 1996, he moved to Public University of Navarra (Pamplona, Spain) where he became a Full Professor in the Electrical and Electronic Engineering department and is currently the head of the optical communications group of this department. He has been Chairman of the Optoelectronic Committee of Spain. He has been leader of more than 50 research projects and he has coauthored more than 250 works in international refereed journals and conferences related with fiber-optic networks, optical amplifiers, fiber-optic sensors, and integrated optics. $\mathrm{He}$ is a member of the technical committees of the International Conference on fiber optic sensors (OFS) and the European Workshop on optical fiber sensors (EWOFS) among others. Professor López-Amo is senior member of the IEEE and member of the OSA. 